Article

\title{
A Few Finite Trigonometric Sums
}

\author{
Chandan Datta ${ }^{1,2, *}$ and Pankaj Agrawal ${ }^{1,2}$ \\ 1 Institute of Physics, Sachivalaya Marg, Bhubaneswar 751005, India; agrawal@iopb.res.in \\ 2 Homi Bhabha National Institute, Training School Complex, Anushakti Nagar, Mumbai 400085, India \\ * Correspondence: chandan@iopb.res.in
}

Academic Editor: Palle E.T. Jorgensen

Received: 30 November 2016; Accepted: 13 February 2017; Published: 18 February 2017

\begin{abstract}
Finite trigonometric sums occur in various branches of physics, mathematics, and their applications. These sums may contain various powers of one or more trigonometric functions. Sums with one trigonometric function are known; however, sums with products of trigonometric functions can become complicated, and may not have a simple expression in a number of cases. Some of these sums have interesting properties, and can have amazingly simple values. However, only some of them are available in the literature. We obtain a number of such sums using the method of residues.
\end{abstract}

Keywords: series sum; complex variables; residue theorem

\section{Introduction}

There is a venerable tradition of computing finite sums of products of trigonometric functions in the literature [1,2]. Such sums occur while addressing many different problems in physics, mathematics, or their applications. Sums such as [1]:

$$
\sum_{j=1}^{d-1} \sin \left(\frac{2 \pi m j}{d}\right) \cot \left(\frac{\pi j}{d}\right)=d-2 m
$$

where $d$ and $m$ are positive integers such that $m<d$, have been known for a long time. However, if a small variation of the arguments of these functions is made, like arguments are affine functions, then these sums no longer remain easy to compute and are not available in various standard handbooks of mathematics [3], including those that specialize in series sum [4-6]. As an example, we may wish to compute:

$$
\sum_{j=1}^{d-1} \sin \left(\frac{2 \pi m j}{d}+a\right) \cot \left(\frac{\pi j}{d}+\pi b\right)
$$

There exist useful sum and difference formulas for sines and cosines that can be used, but such formulas do not exist for other trigonometric functions. In such cases, there is a need to compute sums separately. For example, the above sum can be computed for $b=0$ by using existing results in the handbooks, but for $b \neq 0$, the sum is nontrivial. In this paper, our focus is on such sums.

We have encountered such a sum in analyzing a Bell-type inequality for a system of two finite-dimensional subsystems. We were computing Bell-Son-Lee-Kim (Bell-SLK) function for the general state of a bipartite quantum system. We encountered the following sum [7]:

$$
\sum_{\alpha=0}^{d-1} \cos \left(\frac{2 \pi m}{d}\left(\alpha+\frac{1}{4}\right)\right) \cot \left(\frac{\pi}{d}\left(\alpha+\frac{1}{4}\right)\right)
$$


where $m$ and $d$ are integers satisfying $0<m<d$. We can use the cosine sum rule, but there is no such rule for cotangent. So, we cannot compute it using results given in standard handbooks. This sum can be computed using a corollary, given below. Interestingly, this complicated-looking sum has the value $d$, which is remarkably simple, and is independent of $m$. Independence of $m$ is intriguing, and may have deeper mathematical meaning. Finite trigonometric sums have also appeared in the study of chiral Potts model [8], theory of Dirac operators [9], Dedekind sums [10], theory of determinants and permanents [11,12], and many other places.

There are many techniques for computing these sums; e.g., use of generating functions, Fourier analysis, method of residues, etc. Sometimes, the same sum can be obtained by different methods, giving different-looking results. Chu and Marini [13] have used generating functions extensively to compute trigonometric sums. Berndt and Yeap [14] have used the method of residues. In this work, we will employ the method of residues $[14,15]$. We will start with a function with suitable singularity structure. The function and contour will be chosen in such a way that integration of the function over the contour gives the desired series and its sum. So, the main trick is to find suitable functions and compute residues at poles.

In the next section, we have computed a few sums involving the product of two different trigonometric functions. In Section 3, we generalize the results to the sums of the product of more than two trigonometric functions. In Section 4, we conclude.

\section{Products of Two Trigonometric Functions}

In this section, we will compute the finite sums involving the product of sine with powers of cotangent/cosecants and cosine with powers of cotangent/cosecants. As a byproduct, we will also get a result involving tangent instead of cotangent. In computing these sums, we will use the expansion:

$$
\frac{1}{t \mathrm{e}^{z}-1}=\sum_{v=0}^{\infty} \frac{A_{v}(t)}{v !} z^{v}
$$

where $A_{v}(t)$ is a function of $t$ and $t \neq 1$. The functions $A_{v}(t)$ can be written in terms of the so-called "Apostol-Bernoulli numbers" $B_{v}(0, t)[16]$. In fact:

$$
A_{v}(t)=\frac{B_{v+1}(0, t)}{v+1}
$$

The first few terms are $A_{0}(t)=\frac{1}{t-1}, A_{1}(t)=\frac{-t}{(t-1)^{2}}, A_{2}(t)=\frac{t+t^{2}}{(t-1)^{3}}$, and $A_{3}(t)=\frac{-\left(t+4 t^{2}+t^{3}\right)}{(t-1)^{4}}$. We will also need to expand cotangent in a power series:

$$
\cot (\pi w)=\sum_{j=0}^{\infty} C_{j} \pi^{2 j-1} w^{2 j-1}
$$

where $w$ satisfies $0<|w|<\pi$, and:

$$
C_{j}=\frac{(-1)^{j} 2^{2 j} B_{2 j}}{(2 j) !}
$$

where $B_{j}$ are the well-known "Bernoulli Numbers". The first few $C_{j}$ are $C_{0}=1, C_{1}=-\frac{1}{3}, C_{2}=-\frac{1}{45}$, and $C_{3}=-\frac{2}{945}$. In our case, we need the expansion:

$$
\cot (\pi z+\pi b)=\sum_{j=0}^{\infty} C_{j} \pi^{2 j-1}(z-1+b)^{2 j-1}
$$

with the condition $0<|z-1+b|<\pi$. Let us start with the following theorem. 
Theorem 1. If $m, n$, and denote positive integers with $m<d$ and $b \notin \mathbb{Z} / d$, then:

$$
\begin{gathered}
e_{n}(d, m)=\sum_{j=0}^{d-1} \cos \left(\frac{2 \pi m j}{d}\right) \cot ^{n}\left(\frac{\pi j}{d}+\pi b\right) \\
=-\sum \mathrm{i}^{\mu+v+1} 2^{\mu+v} \frac{m^{\mu}}{\mu !} \frac{d^{v+1}}{v !}\left(t_{1} A_{v}\left(t_{2}\right)-(-1)^{\mu+v} t_{1}^{\prime} A_{v}\left(t_{2}^{\prime}\right)\right) \\
D\left(j_{1}, j_{2}, \ldots, j_{n}\right)
\end{gathered}
$$

Here, the sum is over all nonnegative integers $j_{1}, \ldots, j_{n}, \mu$, and $v$ such that $2 j_{1}+\cdots+2 j_{n}+\mu+v=n-1$. We also have $t_{1}=\mathrm{e}^{-2 \pi \mathrm{i} m b}, t_{2}=\mathrm{e}^{-2 \pi \mathrm{i} d b}, t_{1}^{\prime}=\mathrm{e}^{2 \pi \mathrm{i} m b}$, and $t_{2}^{\prime}=\mathrm{e}^{2 \pi \mathrm{i} d b}$; here, $b \notin \mathbb{Z} / d$ so that the trigonometric sum is well-defined.

Furthermore:

$$
D\left(j_{1}, j_{2}, \ldots, j_{n}\right)=\prod_{r=1}^{n} C_{j_{r}}
$$

Proof. We choose contour $C_{R}$ as a positively-oriented indented rectangle with vertices at $\pm \mathrm{i} R$ and $1 \pm \mathrm{i} R$. The contour has two semicircular indentations of radius $\epsilon(R>\epsilon)$ to the left of both 0 and 1 [14]. Let us take the complex function as:

$$
f(z)=\frac{\mathrm{e}^{2 \pi \mathrm{i} m z} \cot ^{n}(\pi z+\pi b)}{\mathrm{e}^{2 \pi \mathrm{i} d z}-1}-\frac{\mathrm{e}^{-2 \pi \mathrm{i} m z} \cot ^{n}(\pi z+\pi b)}{\mathrm{e}^{-2 \pi \mathrm{i} d z}-1}
$$

and consider $\frac{1}{2 \pi \mathrm{i}} \int_{C} f(z) d z$. Since $f(z)$ has period 1 , the integrals along the indented vertical sides of $C_{R}$ cancel. Since we have taken $m<d, f(z)$ tends to zero uniformly for $0 \leqslant x \leqslant 1$ as $|y| \rightarrow \infty$. Hence, $\frac{1}{2 \pi \mathrm{i}} \int_{C} f(z) d z=0$. We can now calculate the contour integral using Cauchy's residue theorem. The function $f(z)$ has poles at a number of points. To start with, $f(z)$ has a simple pole at $z=0$, with residue:

$$
\operatorname{Res}(f, 0)=\frac{1}{\pi \mathrm{i} d} \cot ^{n}(b \pi)
$$

The function $f(z)$ also has simple poles at $z=\frac{j}{d}$, with $1 \leqslant j \leqslant d-1$. The corresponding residues at these points are:

$$
\operatorname{Res}\left(f, \frac{j}{d}\right)=\frac{1}{\pi \mathrm{i} d} \cos \left(\frac{2 \pi m j}{d}\right) \cot ^{n}\left(\frac{\pi j}{d}+\pi b\right)
$$

In addition, the function $f(z)$ has a pole of order $n$ at $z=-b+1$. Using Equations (4) and (8), we can write:

$$
\begin{aligned}
f(z)= & t_{1} \sum_{\mu=0}^{\infty} \frac{(2 \pi \mathrm{i} m)^{\mu}}{\mu !}(z+b-1)^{\mu}\left(\sum_{j=0}^{\infty} C_{j} \pi^{2 j-1}(z-1+b)^{2 j-1}\right)^{n} \\
& \sum_{v=0}^{\infty} \frac{(2 \pi \mathrm{i} d)^{v}}{v !} A_{v}\left(t_{2}\right)(z+b-1)^{v} \\
- & t_{1}^{\prime} \sum_{\mu=0}^{\infty} \frac{(-2 \pi \mathrm{i} m)^{\mu}}{\mu !}(z+b-1)^{\mu}\left(\sum_{j=0}^{\infty} C_{j} \pi^{2 j-1}(z-1+b)^{2 j-1}\right)^{n} \\
& \sum_{v=0}^{\infty} \frac{(-2 \pi \mathrm{i} d)^{v}}{v !} A_{v}\left(t_{2}^{\prime}\right)(z+b-1)^{v}
\end{aligned}
$$

Then, after few steps of straightforward calculation, one can show that:

$$
\operatorname{Res}(f,-b+1)=\sum \mathrm{i}^{\mu+v} 2^{\mu+v} \frac{m^{\mu}}{\mu !} \frac{d^{v}}{v !}\left(\frac{t_{1}}{\pi} A_{v}\left(t_{2}\right)-(-1)^{\mu+v} \frac{t_{1}^{\prime}}{\pi} A_{v}\left(t_{2}^{\prime}\right)\right) D\left(j_{1}, j_{2}, \ldots, j_{n}\right)
$$


Here, the sum is over all nonnegative integers $j_{1}, \ldots, j_{n}, \mu$, and $v$ such that $2 j_{1}+\cdots+2 j_{n}+\mu+v=$ $n-1$. Using (12), (13), (15), and applying residue theorem, we can obtain the sum (9).

Corollary 1. Let $m$ and $d$ be positive integers such that $m<d$ and $b \notin \mathbb{Z} / d$. Then:

$$
e_{1}(d, m)=d \cos [(2 m-d) b \pi] \operatorname{cosec}(b d \pi)
$$

Proof. Put $n=1$ in Theorem 1 . Using the values $A_{0}(t)$ and $C_{0}$, one can easily see this.

We will now consider sums involving sine and cotangents. We will need to modify our functions suitably.

Theorem 2. Let $m, n$, and $d$ denote positive integers with $m<d$ and $b \notin \mathbb{Z} / d$. Then:

$$
\begin{gathered}
g_{n}(d, m)=-\sum \mathrm{i}^{\mu+v} 2^{\mu+v} \frac{m^{\mu}}{\mu !} \frac{d^{v+1}}{v !}\left(t_{1} A_{v}\left(t_{2}\right)+(-1)^{\mu+v} t_{1}^{\prime} A_{v}\left(t_{2}^{\prime}\right)\right) \\
D\left(j_{1}, j_{2}, \ldots, j_{n}\right)
\end{gathered}
$$

We have already defined all the terms and conditions in Theorem 1. Here:

$$
g_{n}(d, m)=\sum_{j=1}^{d-1} \sin \left(\frac{2 \pi m j}{d}\right) \cot ^{n}\left(\frac{\pi j}{d}+\pi b\right)
$$

Proof. Our contour will be the same as in Theorem 1. Let us take the complex function as:

$$
f(z)=\frac{\mathrm{e}^{2 \pi \mathrm{i} m z} \cot ^{n}(\pi z+\pi b)}{\mathrm{e}^{2 \pi \mathrm{i} d z}-1}+\frac{\mathrm{e}^{-2 \pi \mathrm{i} m z} \cot ^{n}(\pi z+\pi b)}{\mathrm{e}^{-2 \pi \mathrm{i} d z}-1}
$$

and consider $\frac{1}{2 \pi \mathrm{i}} \int_{C} f(z) d z$. As before, $\int_{C} f(z) d z=0$. The pole structure of this function is the same as in Theorem 1. However, this time residue is zero at $z=0$, so we take the sum from $j=1$. Since $\sin x$ vanishes at $x=0$, we can take the sum from $j=0$. The function $f(z)$ has simple poles at $z=\frac{j}{d}$, with $1 \leqslant j \leqslant d-1$. The corresponding residues at these points are:

$$
\operatorname{Res}\left(f, \frac{j}{d}\right)=\frac{1}{\pi d} \sin \left(\frac{2 \pi m j}{d}\right) \cot ^{n}\left(\frac{\pi j}{d}+\pi b\right)
$$

The function $f(z)$ also has a pole of order $n$ at $z=-b+1$. Using (4) and (8) (as in the case of the last theorem), after a few steps of straight forward calculation, we can obtain:

$$
\operatorname{Res}(f,-b+1)=\sum \mathrm{i}^{\mu+v} 2^{\mu+v} \frac{m^{\mu}}{\mu !} \frac{d^{v}}{v !}\left(\frac{t_{1}}{\pi} A_{v}\left(t_{2}\right)+(-1)^{\mu+v} \frac{t_{1}^{\prime}}{\pi} A_{v}\left(t_{2}^{\prime}\right)\right) D\left(j_{1}, j_{2}, \ldots, j_{n}\right)
$$

Using (20) and (21), and applying residue theorem, we can easily obtain (17).

Corollary 2. Let $m$ and $d$ be positive integers such that $m<d$ and $b \notin \mathbb{Z} / d$. Then:

$$
g_{1}(d, m)=-d \sin [(2 m-d) b \pi] \operatorname{cosec}(b d \pi)
$$

Proof. Put $n=1$ in Theorem 2. Using the values $A_{0}(t)$ and $C_{0}$, we can obtain this.

We will now consider sums involving sine and cosecants. We will do this for even and odd powers of cosecants separately. 
Theorem 3. Let $m, n$, and $d$ denote positive integers with $m<d$ and $b \notin \mathbb{Z} / d$. Then:

$$
\begin{gathered}
h_{n}(d, m)=-\sum \mathrm{i}^{\mu+v} 2^{\mu+v} \frac{m^{\mu}}{\mu !} \frac{d^{v+1}}{v !}\left(t_{1} A_{v}\left(t_{2}\right)+(-1)^{\mu+v} t_{1}^{\prime} A_{v}\left(t_{2}^{\prime}\right)\right) \\
F\left(j_{1}, j_{2}, \ldots, j_{2 n}\right)
\end{gathered}
$$

where the sum is over all nonnegative integers $j_{1}, \ldots, j_{2 n}, \mu$, and $v$ such that $2 j_{1}+\cdots+2 j_{2 n}+\mu+v=2 n-1$ and $\mu+v$ must be odd. All other terms have already been defined in Theorem 1 , except for $F\left(j_{1}, j_{2}, \ldots, j_{2 n}\right)$. Here:

$$
h_{n}(d, m)=\sum_{j=1}^{d-1} \sin \left(\frac{2 \pi m j}{d}\right) \operatorname{cosec}^{2 n}\left(\frac{\pi j}{d}+\pi b\right)
$$

The function $F\left(j_{1}, j_{2}, \ldots, j_{2 n}\right)$ is defined using the expansion:

$$
\operatorname{cosec}(\pi z+\pi b)=-\operatorname{cosec}(\pi z+\pi b-\pi)=\sum_{j=0}^{\infty} G_{j} \pi^{2 j-1}(z-1+b)^{2 j-1}
$$

with $0<|z-1+b|<\pi$. Here:

$$
G_{j}=\frac{(-1)^{j 2}\left(2^{2 j-1}-1\right) B_{2 j}}{(2 j) !}
$$

where $B_{j}$ is Bernoulli number. The first few $G_{j}$ are $G_{0}=-1, G_{1}=-\frac{1}{6}, G_{2}=-\frac{7}{360}$, and $G_{3}=-\frac{31}{15120}$. Then:

$$
F\left(j_{1}, j_{2}, \ldots, j_{2 n}\right)=\prod_{r=1}^{2 n} G_{j_{r}}
$$

Proof. Let us take the complex function as:

$$
f(z)=\frac{\mathrm{e}^{2 \pi \mathrm{i} m z} \operatorname{cosec}^{2 n}(\pi z+\pi b)}{\mathrm{e}^{2 \pi \mathrm{i} d z}-1}+\frac{\mathrm{e}^{-2 \pi \mathrm{i} m z} \operatorname{cosec}^{2 n}(\pi z+\pi b)}{\mathrm{e}^{-2 \pi \mathrm{i} d z}-1}
$$

We can use the same contour and follow the same procedure as in Theorems 1 and 2 . The function $f(z)$ has simple poles at $z=\frac{j}{d}$, with $1 \leqslant j \leqslant d-1$ with residues:

$$
\operatorname{Res}\left(f, \frac{j}{d}\right)=\frac{1}{\pi d} \sin \left(\frac{2 \pi m j}{d}\right) \operatorname{cosec}^{2 n}\left(\frac{\pi j}{d}+\pi b\right)
$$

The function $f(z)$ also has a pole of order $2 n$ at $z=-b+1$. Using (4) and (25), we can write:

$$
\begin{aligned}
f(z)= & t_{1} \sum_{\mu=0}^{\infty} \frac{(2 \pi \mathrm{i} m)^{\mu}}{\mu !}(z+b-1)^{\mu}\left(\sum_{j=0}^{\infty} B_{j} \pi^{2 j-1}(z-1+b)^{2 j-1}\right)^{2 n} \\
& \sum_{v=0}^{\infty} \frac{(2 \pi \mathrm{i} d)^{v}}{v !} A_{v}\left(t_{2}\right)(z+b-1)^{v} \\
+ & t_{1}^{\prime} \sum_{\mu=0}^{\infty} \frac{(-2 \pi \mathrm{i} m)^{\mu}}{\mu !}(z+b-1)^{\mu}\left(\sum_{j=0}^{\infty} B_{j} \pi^{2 j-1}(z-1+b)^{2 j-1}\right)^{2 n} \\
& \sum_{v=0}^{\infty} \frac{(-2 \pi \mathrm{i} d)^{v}}{v !} A_{v}\left(t_{2}^{\prime}\right)(z+b-1)^{v}
\end{aligned}
$$

After a few steps of straightforward calculation, one obtains:

$$
\operatorname{Res}(f,-b+1)=\sum \mathrm{i}^{\mu+v} 2^{\mu+v} \frac{m^{\mu}}{\mu !} \frac{d^{v}}{v !}\left(\frac{t_{1}}{\pi} A_{v}\left(t_{2}\right)+(-1)^{\mu+v} \frac{t_{1}^{\prime}}{\pi} A_{v}\left(t_{2}^{\prime}\right)\right) F\left(j_{1}, j_{2}, \ldots, j_{n}\right)
$$


Using (29), (31), and applying residue theorem, we can easily obtain (23).

Corollary 3. Let $m$ and $d$ be positive integers, such that $m<d$ and $b \notin \mathbb{Z} / d$. Then:

$$
h_{1}(d, m)=d \operatorname{cosec}^{2}((b-1) d \pi)[m \sin (2(b-1)(d-m) \pi)-(d-m) \sin (2(b-1) m \pi)]
$$

Proof. Put $n=1$ in Theorem 3. Using the values $A_{0}(t), A_{1}(t)$ and $B_{0}$, one can show this.

We will next consider a sum involving cosine and even powers of the cosecant. We will only have to change the function slightly.

Theorem 4. Let $m, n$, and $d$ denote positive integers with $m<d$ and $b \notin \mathbb{Z} / d$. Then:

$$
\begin{gathered}
k_{n}(d, m)=-\sum \mathrm{i}^{\mu+v+1} 2^{\mu+v} \frac{m^{\mu}}{\mu !} \frac{d^{v+1}}{v !}\left(t_{1} A_{v}\left(t_{2}\right)-(-1)^{\mu+v} t_{1}^{\prime} A_{v}\left(t_{2}^{\prime}\right)\right) \\
F\left(j_{1}, j_{2}, \ldots, j_{2 n}\right)
\end{gathered}
$$

where the sum is over all nonnegative integers $j_{1}, \ldots, j_{2 n}, \mu$, and $v$ such that $2 j_{1}+\cdots+2 j_{2 n}+\mu+v=2 n-1$ and $\mu+v+1$ must be even. Here:

$$
k_{n}(d, m)=\sum_{j=0}^{d-1} \cos \left(\frac{2 \pi m j}{d}\right) \operatorname{cosec}^{2 n}\left(\frac{\pi j}{d}+\pi b\right)
$$

Proof. Let us take the complex function as:

$$
f(z)=\frac{\mathrm{e}^{2 \pi \mathrm{i} m z} \operatorname{cosec}^{2 n}(\pi z+\pi b)}{\mathrm{e}^{2 \pi \mathrm{i} d z}-1}-\frac{\mathrm{e}^{-2 \pi \mathrm{i} m z} \operatorname{cosec}^{2 n}(\pi z+\pi b)}{\mathrm{e}^{-2 \pi \mathrm{i} d z}-1}
$$

Then, applying the same procedure as in Theorem 3, we can obtain (33).

Corollary 4. Let $m$ and $d$ be positive integers such that $m<d$ and $b \notin \mathbb{Z} / d$. Then:

$$
k_{1}(d, m)=d \operatorname{cosec}^{2}((b-1) d \pi)[m \cos (2(b-1)(d-m) \pi)+(d-m) \cos (2(b-1) m \pi)]
$$

Proof. Put $n=1$ in Theorem 4. Using the values $A_{0}(t), A_{1}(t)$, and $B_{0}$, this result can be obtained.

Let us now consider odd powers of cosecants. In this case, to keep the periodicity of the function $f(z)$, we shall have to use the argument of sine as $\frac{\pi m j}{d}$, instead of $\frac{2 \pi m j}{d}$ as earlier.

Theorem 5. If $m$ is an odd positive integer, and $n$ and $d$ are positive integers with $m<d$, then:

$$
\begin{gathered}
l_{n}(d, m)=-\sum \mathrm{i}^{\mu+v} 2^{v} \frac{m^{\mu}}{\mu !} \frac{d^{v+1}}{v !}\left(p_{1} A_{v}\left(p_{2}\right)+(-1)^{\mu+v} p_{1}^{\prime} A_{v}\left(p_{2}^{\prime}\right)\right) \\
F\left(j_{1}, j_{2}, \ldots, j_{2 n-1}\right)
\end{gathered}
$$

where the sum is over all nonnegative integers $j_{1}, \ldots, j_{2 n-1}, \mu$, and $v$ such that $2 j_{1}+\cdots+2 j_{2 n-1}+\mu+v=$ $2 n-2$, and $\mu+v$ must be even. Here, $p_{1}=\mathrm{e}^{-\pi \mathrm{i} m(b-1)}, p_{2}=\mathrm{e}^{-2 \pi \mathrm{i} d b}, p_{1}^{\prime}=\mathrm{e}^{\pi \mathrm{i} m(b-1)}$, and $p_{2}^{\prime}=\mathrm{e}^{2 \pi \mathrm{i} d b}$; $b \notin \mathbb{Z} / d$ such that the trigonometric sum is well-defined:

$$
l_{n}(d, m)=\sum_{j=1}^{d-1} \sin \left(\frac{\pi m j}{d}\right) \operatorname{cosec}^{2 n-1}\left(\frac{\pi j}{d}+\pi b\right)
$$


Proof. Let us take the complex function as:

$$
f(z)=\frac{\mathrm{e}^{\pi \mathrm{i} m z} \operatorname{cosec}^{2 n-1}(\pi z+\pi b)}{\mathrm{e}^{2 \pi \mathrm{i} d z}-1}+\frac{\mathrm{e}^{-\pi \mathrm{i} m z} \operatorname{cosec}^{2 n-1}(\pi z+\pi b)}{\mathrm{e}^{-2 \pi \mathrm{i} d z}-1}
$$

Proceeding in the same way as before, we can obtain (37).

Corollary 5. If $m$ is an odd positive integer, and $n$ and $d$ are positive integers with $m<d$ and $b \notin \mathbb{Z} / d$, then:

$$
l_{1}(d, m)=-d \sin ((m-d) b \pi) \operatorname{cosec}(\pi d b)
$$

Proof. By putting $n=1$ in Theorem 5 and taking the values of $A_{0}(p)$ and $B_{0}$, one can easily see this.

Again as before, in the above sum, sine can be replaced with cosine by modifying $f(z)$ slightly.

Theorem 6. If $m$ is an positive odd integer, and $n$ and $d$ are positive integers with $m<d$ and $b \notin \mathbb{Z} / d$, then:

$$
\begin{gathered}
q_{n}(d, m)=-\sum \mathrm{i}^{\mu+v+1} 2^{v} \frac{m^{\mu}}{\mu !} \frac{d^{v+1}}{v !}\left(p_{1} A_{v}\left(p_{2}\right)-(-1)^{\mu+v} p_{1}^{\prime} A_{v}\left(p_{2}^{\prime}\right)\right) \\
F\left(j_{1}, j_{2}, \ldots, j_{2 n-1}\right)
\end{gathered}
$$

where the sum is over all nonnegative integers $j_{1}, \ldots, j_{2 n-1}, \mu$, and $v$ such that $2 j_{1}+\cdots+2 j_{2 n-1}+\mu+v=$ $2 n-2$ and $\mu+v$ must be even. Here:

$$
q_{n}(d, m)=\sum_{j=0}^{d-1} \cos \left(\frac{\pi m j}{d}\right) \operatorname{cosec}^{2 n-1}\left(\frac{\pi j}{d}+\pi b\right)
$$

Proof. Let us take the complex function as:

$$
f(z)=\frac{\mathrm{e}^{\pi \mathrm{i} m z} \operatorname{cosec}^{2 n-1}(\pi z+\pi b)}{\mathrm{e}^{2 \pi \mathrm{i} d z}-1}-\frac{\mathrm{e}^{-\pi \mathrm{i} m z} \operatorname{cosec}^{2 n-1}(\pi z+\pi b)}{\mathrm{e}^{-2 \pi \mathrm{i} d z}-1}
$$

Proceeding in the same way as before, we can obtain (41).

Corollary 6. If $m$ is an odd positive integer, and $n$ and $d$ are positive integers with $m<d$ and $b \notin \mathbb{Z} / d$, then:

$$
q_{1}(d, m)=d \cos ((m-d) b \pi) \operatorname{cosec}(\pi d b)
$$

Proof. By putting $n=1$ in Theorem 6 and taking the values of $A_{0}(p)$ and $B_{0}$, we can easily obtain this.

If we take tangent instead of cotangent in the sum of Corollary 1 , then we will get:

$$
\sum_{j=0}^{d-1} \cos \left(\frac{2 \pi m j}{d}\right) \tan \left(\frac{\pi j}{d}+\pi b\right)= \begin{cases}(-1)^{m+1} d \cos [(2 m-d) b \pi] \operatorname{cosec}(b d \pi) ; & \text { if } d=\text { even } \\ (-1)^{m-d} d \sin [(2 m-d) b \pi] \sec (b d \pi) ; & \text { if } d=\text { odd }\end{cases}
$$

Similarly, if we take tangent instead of cotangent in the sum of Corollary 2, then:

$$
\sum_{j=1}^{d-1} \sin \left(\frac{2 \pi m j}{d}\right) \tan \left(\frac{\pi j}{d}+\pi b\right)= \begin{cases}(-1)^{m} d \sin [(2 m-d) b \pi] \operatorname{cosec}(b d \pi) ; & \text { if } d=\text { even } \\ (-1)^{m-d} d \cos [(2 m-d) b \pi] \sec (b d \pi) ; & \text { if } d=\text { odd }\end{cases}
$$

For even $d$, the sums in (45) and (46) are not defined when $b \in \mathbb{Z} / d$, and for odd $d$, the sums are not defined when $b \in \mathbb{O} / 2 d$, where $\mathbb{O}$ is the set of odd integers. 
Many more sums of the product of two trigonometric functions can be obtained with the same contour, with a change of function. As an example, if we slightly change the arguments of the exponentials of the complex functions $f(z)$, we can get a different kind of sums. To illustrate this, we can take the factor of 4 instead of 2 in the exponentials of the function $f(z)$ in Theorems 1 and 2 . Following the same procedure as used there, we get:

$$
\sum_{j=0}^{2 d-1} \cos \left(\frac{2 \pi m j}{d}\right) \cot \left(\frac{\pi j}{2 d}+\pi b\right)=2 d \cos [(2 m-d) 2 b \pi] \operatorname{cosec}(2 b d \pi)
$$

and:

$$
\sum_{j=1}^{2 d-1} \sin \left(\frac{2 \pi m j}{d}\right) \cot \left(\frac{\pi j}{2 d}+\pi b\right)=-2 d \sin [(2 m-d) 2 b \pi] \operatorname{cosec}(2 b d \pi)
$$

respectively, and $b \notin \mathbb{Z} / 2 d$ such that the sum is well defined.

These are just a sample of sums that one can obtain with the contour described in Theorem 1 , but choosing a variety of integrands.

\section{Products of More than Two Trigonometric Functions}

We shall now consider a few sums involving the products of three trigonometric functions. We shall consider only those functions for which integral along the two sides of the contour cancel each other. Let us consider the following function:

$$
f(z)=\left[\frac{\mathrm{e}^{2 \pi \mathrm{i} m z}}{\mathrm{e}^{2 \pi \mathrm{i} d z}-1}-\frac{\mathrm{e}^{-2 \pi \mathrm{i} m z}}{\mathrm{e}^{-2 \pi \mathrm{i} d z}-1}\right] \operatorname{cosec}\left(\pi z+\pi b_{1}\right) \cos \left(\pi z+\pi b_{2}\right)
$$

Using the same contour and the same procedure as in Theorems 1 and 2, we get:

$$
\begin{aligned}
& \sum_{j=0}^{d-1} \cos \left(\frac{2 \pi m j}{d}\right) \operatorname{cosec}\left(\frac{\pi j}{d}+\pi b_{1}\right) \cos \left(\frac{\pi j}{d}+\pi b_{2}\right) \\
= & -d \cos \left[(2 m-d) b_{1} \pi\right] \operatorname{cosec}\left(b_{1} d \pi\right) \cos \left[\pi+\left(b_{2}-b_{1}\right) \pi\right]
\end{aligned}
$$

In the same way, by replacing the last term by sine in (49), we get:

$$
\begin{aligned}
& \sum_{j=0}^{d-1} \cos \left(\frac{2 \pi m j}{d}\right) \operatorname{cosec}\left(\frac{\pi j}{d}+\pi b_{1}\right) \sin \left(\frac{\pi j}{d}+\pi b_{2}\right) \\
= & -d \cos \left[(2 m-d) b_{1} \pi\right] \operatorname{cosec}\left(b_{1} d \pi\right) \sin \left[\pi+\left(b_{2}-b_{1}\right) \pi\right]
\end{aligned}
$$

Of course, this sum (as before) is valid when $b_{1} \notin \mathbb{Z} / d$ such that the trigonometric sum is well defined. By replacing the cosecant in (49) by secant, we get following two sums:

$$
\sum_{j=0}^{d-1} \cos \left(\frac{2 \pi m j}{d}\right) \sec \left(\frac{\pi j}{d}+\pi b_{1}\right) \cos \left(\frac{\pi j}{d}+\pi b_{2}\right)= \begin{cases}(-1)^{m+1} d \cos \left[(2 m-d) b_{1} \pi\right] \operatorname{cosec}\left(b_{1} d \pi\right) \\ \cos \left[\frac{\pi}{2}+\left(b_{2}-b_{1}\right) \pi\right] ; & \text { if } d=\text { even } \\ (-1)^{m-d} d \sin \left[(2 m-d) b_{1} \pi\right] \sec \left(b_{1} d \pi\right) & \text { if } d=\text { odd } \\ \cos \left[\frac{\pi}{2}+\left(b_{2}-b_{1}\right) \pi\right] ; & \end{cases}
$$

and:

$$
\sum_{j=0}^{d-1} \cos \left(\frac{2 \pi m j}{d}\right) \sec \left(\frac{\pi j}{d}+\pi b_{1}\right) \sin \left(\frac{\pi j}{d}+\pi b_{2}\right)= \begin{cases}(-1)^{m+1} d \cos \left[(2 m-d) b_{1} \pi\right] \operatorname{cosec}\left(b_{1} d \pi\right) \\ \sin \left[\frac{\pi}{2}+\left(b_{2}-b_{1}\right) \pi\right] ; & \text { if } d=\text { even } \\ (-1)^{m-d} d \sin \left[(2 m-d) b_{1} \pi\right] \sec \left(b_{1} d \pi\right) & \text { if } d=\text { odd }\end{cases}
$$


For even $d$, the sums are well defined if $b_{1} \notin \mathbb{Z} / d$, and for odd $d$ the condition on $b_{1}$ is $b_{1} \notin \mathbb{O} / 2 d$.

In function (49), if we replace the minus sign between two exponentials with a plus sign, we will find:

$$
\begin{aligned}
& \sum_{j=1}^{d-1} \sin \left(\frac{2 \pi m j}{d}\right) \operatorname{cosec}\left(\frac{\pi j}{d}+\pi b_{1}\right) \cos \left(\frac{\pi j}{d}+\pi b_{2}\right) \\
& =d \sin \left[(2 m-d) b_{1} \pi\right] \operatorname{cosec}\left(b_{1} d \pi\right) \cos \left[\pi+\left(b_{2}-b_{1}\right) \pi\right]
\end{aligned}
$$

Similarly, replacing the last cosine term by sine, we get:

$$
\begin{aligned}
& \sum_{j=1}^{d-1} \sin \left(\frac{2 \pi m j}{d}\right) \operatorname{cosec}\left(\frac{\pi j}{d}+\pi b_{1}\right) \sin \left(\frac{\pi j}{d}+\pi b_{2}\right) \\
& =d \sin \left[(2 m-d) b_{1} \pi\right] \operatorname{cosec}\left(b_{1} d \pi\right) \sin \left[\pi+\left(b_{2}-b_{1}\right) \pi\right]
\end{aligned}
$$

The condition on $b_{1}$ for the above two sums is $b_{1} \notin \mathbb{Z} / d$.

Just like we obtained (52) and (53), we can find:

$$
\sum_{j=1}^{d-1} \sin \left(\frac{2 \pi m j}{d}\right) \sec \left(\frac{\pi j}{d}+\pi b_{1}\right) \cos \left(\frac{\pi j}{d}+\pi b_{2}\right)= \begin{cases}(-1)^{m} d \sin \left[(2 m-d) b_{1} \pi\right] \operatorname{cosec}\left(b_{1} d \pi\right) \\ \cos \left[\frac{\pi}{2}+\left(b_{2}-b_{1}\right) \pi\right] ; & \text { if } d=\text { even } \\ (-1)^{m-d} d \cos \left[(2 m-d) b_{1} \pi\right] \sec \left(b_{1} d \pi\right) & \text { if } d=\text { odd }\end{cases}
$$

and:

$$
\sum_{j=1}^{d-1} \sin \left(\frac{2 \pi m j}{d}\right) \sec \left(\frac{\pi j}{d}+\pi b_{1}\right) \sin \left(\frac{\pi j}{d}+\pi b_{2}\right)= \begin{cases}(-1)^{m} d \sin \left[(2 m-d) b_{1} \pi\right] \operatorname{cosec}\left(b_{1} d \pi\right) \\ \sin \left[\frac{\pi}{2}+\left(b_{2}-b_{1}\right) \pi\right] ; & \text { if } d=\text { even } \\ (-1)^{m-d} d \cos \left[(2 m-d) b_{1} \pi\right] \sec \left(b_{1} d \pi\right) & \text { if } d=\text { odd }\end{cases}
$$

For even $d$, the above two sums are well-defined if $b_{1} \notin \mathbb{Z} / d$ and for odd $d$, the condition is $b_{1} \notin \mathbb{O} / 2 d$.

Let us now take a different complex function. We can then obtain many more sums:

$$
f(z)=\left[\frac{\mathrm{e}^{2 \pi \mathrm{i} m z}}{\mathrm{e}^{2 \pi \mathrm{i} d z}-1}-\frac{\mathrm{e}^{-2 \pi \mathrm{i} m z}}{\mathrm{e}^{-2 \pi \mathrm{i} d z}-1}\right] \operatorname{cosec}\left(\pi z+\pi b_{1}\right) \operatorname{cosec}\left(\pi z+\pi b_{2}\right)
$$

By using residue theorem, we can show that:

$$
\begin{aligned}
& \sum_{j=0}^{d-1} \cos \left(\frac{2 \pi m j}{d}\right) \operatorname{cosec}\left(\frac{\pi j}{d}+\pi b_{1}\right) \operatorname{cosec}\left(\frac{\pi j}{d}+\pi b_{2}\right) \\
& =-d \cos \left[(2 m-d) b_{1} \pi\right] \operatorname{cosec}\left(b_{1} d \pi\right) \operatorname{cosec}\left[\pi+\left(b_{2}-b_{1}\right) \pi\right] \\
& \quad-d \cos \left[(2 m-d) b_{2} \pi\right] \operatorname{cosec}\left(b_{2} d \pi\right) \operatorname{cosec}\left[\pi+\left(b_{1}-b_{2}\right) \pi\right]
\end{aligned}
$$


This sum is valid for $b_{1} \notin \mathbb{Z} / d$ and $b_{2} \notin \mathbb{Z} / d$ such that the trigonometric sum is well defined. In (58) if we use one cosecant and one secant instead of two cosecant, we will get:

$$
\sum_{j=0}^{d-1} \cos \left(\frac{2 \pi m j}{d}\right) \operatorname{cosec}\left(\frac{\pi j}{d}+\pi b_{1}\right) \sec \left(\frac{\pi j}{d}+\pi b_{2}\right)=\left\{\begin{array}{l}
\left(-d \cos \left[(2 m-d) b_{1} \pi\right] \operatorname{cosec}\left(b_{1} d \pi\right)\right. \\
\sec \left[\pi+\left(b_{2}-b_{1}\right) \pi\right]+(-1)^{m+1} d \cos \left[(2 m-d) b_{2} \pi\right] \\
\left.\operatorname{cosec}\left(b_{2} d \pi\right) \operatorname{cosec}\left[\frac{\pi}{2}+\left(b_{1}-b_{2}\right) \pi\right]\right) ; \quad \text { if } d=\text { even } \\
\left(-d \cos \left[(2 m-d) b_{1} \pi\right] \operatorname{cosec}\left(b_{1} d \pi\right)\right. \\
\sec \left[\pi+\left(b_{2}-b_{1}\right) \pi\right]+(-1)^{m-d} d \sin \left[(2 m-d) b_{2} \pi\right] \\
\left.\sec \left(b_{2} d \pi\right) \operatorname{cosec}\left[\frac{\pi}{2}+\left(b_{1}-b_{2}\right) \pi\right]\right) ; \quad \text { if } d=\text { odd }
\end{array}\right.
$$

For even $d$, the above sum is valid for $b_{1} \notin \mathbb{Z} / d$ and $b_{2} \notin \mathbb{Z} / d$. In case of odd $d$, the conditions are $b_{1} \notin \mathbb{Z} / d$ and $b_{2} \notin \mathbb{O} / 2 d$. Again if both are secants, then:

$$
\sum_{j=0}^{d-1} \cos \left(\frac{2 \pi m j}{d}\right) \sec \left(\frac{\pi j}{d}+\pi b_{1}\right) \sec \left(\frac{\pi j}{d}+\pi b_{2}\right)=\left\{\begin{array}{l}
\left((-1)^{m+1} d \cos \left[(2 m-d) b_{1} \pi\right] \operatorname{cosec}\left(b_{1} d \pi\right)\right. \\
\sec \left[\frac{\pi}{2}+\left(b_{2}-b_{1}\right) \pi\right]+(-1)^{m+1} d \cos \left[(2 m-d) b_{2} \pi\right] \\
\left.\operatorname{cosec}\left(b_{2} d \pi\right) \sec \left[\frac{\pi}{2}+\left(b_{1}-b_{2}\right) \pi\right]\right) ; \quad \text { if } d=\text { even } \\
\left((-1)^{m-d} d \sin \left[(2 m-d) b_{1} \pi\right] \sec \left(b_{1} d \pi\right)\right. \\
\sec \left[\frac{\pi}{2}+\left(b_{2}-b_{1}\right) \pi\right]+(-1)^{m-d} d \sin \left[(2 m-d) b_{2} \pi\right] \\
\left.\sec \left(b_{2} d \pi\right) \sec \left[\frac{\pi}{2}+\left(b_{1}-b_{2}\right) \pi\right]\right) ; \quad \text { if } d=\text { odd }
\end{array}\right.
$$

The conditions on $b$ s for even $d$ are $b_{1} \notin \mathbb{Z} / d$ and $b_{2} \notin \mathbb{Z} / d$. For the odd $d$ case, the conditions are $b_{1} \notin \mathbb{O} / 2 d$ and $b_{2} \notin \mathbb{O} / 2 d$.

Like (59)-(61), we can get similar sums with sine as follows:

$$
\begin{aligned}
& \sum_{j=1}^{d-1} \sin \left(\frac{2 \pi m j}{d}\right) \operatorname{cosec}\left(\frac{\pi j}{d}+\pi b_{1}\right) \operatorname{cosec}\left(\frac{\pi j}{d}+\pi b_{2}\right) \\
& =d \sin \left[(2 m-d) b_{1} \pi\right] \operatorname{cosec}\left(b_{1} d \pi\right) \operatorname{cosec}\left[\pi+\left(b_{2}-b_{1}\right) \pi\right] \\
& \quad+d \sin \left[(2 m-d) b_{2} \pi\right] \operatorname{cosec}\left(b_{2} d \pi\right) \operatorname{cosec}\left[\pi+\left(b_{1}-b_{2}\right) \pi\right]
\end{aligned}
$$

Conditions on $b_{1}$ and $b_{2}$ are $b_{1} \notin \mathbb{Z} / d$ and $b_{2} \notin \mathbb{Z} / d$ :

$$
\sum_{j=1}^{d-1} \sin \left(\frac{2 \pi m j}{d}\right) \operatorname{cosec}\left(\frac{\pi j}{d}+\pi b_{1}\right) \sec \left(\frac{\pi j}{d}+\pi b_{2}\right)=\left\{\begin{array}{l}
\left(d \sin \left[(2 m-d) b_{1} \pi\right] \operatorname{cosec}\left(b_{1} d \pi\right)\right. \\
\sec \left[\pi+\left(b_{2}-b_{1}\right) \pi\right]+(-1)^{m} d \sin \left[(2 m-d) b_{2} \pi\right] \\
\left.\operatorname{cosec}\left(b_{2} d \pi\right) \operatorname{cosec}\left[\frac{\pi}{2}+\left(b_{1}-b_{2}\right) \pi\right]\right) ; \quad \text { if } d=\text { even } \\
\left(d \sin \left[(2 m-d) b_{1} \pi\right] \operatorname{cosec}\left(b_{1} d \pi\right)\right. \\
\sec \left[\pi+\left(b_{2}-b_{1}\right) \pi\right]+(-1)^{m-d} d \cos \left[(2 m-d) b_{2} \pi\right] \\
\left.\sec \left(b_{2} d \pi\right) \operatorname{cosec}\left[\frac{\pi}{2}+\left(b_{1}-b_{2}\right) \pi\right]\right) ; \quad \text { if } d=\text { odd }
\end{array}\right.
$$

For even $d$, the above sum is valid for $b_{1} \notin \mathbb{Z} / d$ and $b_{2} \notin \mathbb{Z} / d$. In the case of odd $d$, the conditions are $b_{1} \notin \mathbb{Z} / d$ and $b_{2} \notin \mathbb{O} / 2 d$ :

$$
\sum_{j=0}^{d-1} \sin \left(\frac{2 \pi m j}{d}\right) \sec \left(\frac{\pi j}{d}+\pi b_{1}\right) \sec \left(\frac{\pi j}{d}+\pi b_{2}\right)=\left\{\begin{array}{l}
\left((-1)^{m} d \sin \left[(2 m-d) b_{1} \pi\right] \operatorname{cosec}\left(b_{1} d \pi\right)\right. \\
\sec \left[\frac{\pi}{2}+\left(b_{2}-b_{1}\right) \pi\right]+(-1)^{m} d \sin \left[(2 m-d) b_{2} \pi\right] \\
\left.\operatorname{cosec}\left(b_{2} d \pi\right) \sec \left[\frac{\pi}{2}+\left(b_{1}-b_{2}\right) \pi\right]\right) ; \quad \text { if } d=\text { even } \\
\left((-1)^{m-d} d \cos \left[(2 m-d) b_{1} \pi\right] \sec \left(b_{1} d \pi\right)\right. \\
\sec \left[\frac{\pi}{2}+\left(b_{2}-b_{1}\right) \pi\right]+(-1)^{m-d} d \cos \left[(2 m-d) b_{2} \pi\right] \\
\left.\sec \left(b_{2} d \pi\right) \sec \left[\frac{\pi}{2}+\left(b_{1}-b_{2}\right) \pi\right]\right) ; \quad \text { if } d=\text { odd }
\end{array}\right.
$$


The conditions on $b$ s for even $d$ are $b_{1} \notin \mathbb{Z} / d$ and $b_{2} \notin \mathbb{Z} / d$. For the odd $d$ case, the conditions are $b_{1} \notin \mathbb{O} / 2 d$ and $b_{2} \notin \mathbb{O} / 2 d$.

In this section, we have considered the product of three trigonometric functions with simple powers. One can extend these results to higher powers of trigonometric functions, as well as products of more than three trigonometric functions. The list is endless. We have only illustrated a few cases.

\section{Conclusions}

We have obtained a number of finite sums involving products of two or more trigonometric functions. They were mostly based on a specific choice of contour and a wide variety of integrands. Many more sums can be obtained. For the simpler powers of trigonometric functions, these sums can be remarkably simple. Most of these sums involve tangents, cotangents, secants, and cosecants. For these functions, we do not have simple expansions for the sum and difference of variables in their arguments. Therefore, one has to compute them independently. We have calculated the sums for a few cases. One can extend these calculations in many different directions, as discussed in the above sections. Most of the simpler expressions should be in handbooks that have trigonometric sums.

Author Contributions: Both the authors contributed to the development and design of the problem. Chandan Datta did most of the calculation. All authors contributed to the preparation of the manuscript.

Conflicts of Interest: The authors declare no conflict of interest.

\section{References}

1. Eisenstein, G. Aufgaben und Lehrsätze. J. Reine Angew. Math. 1844, 27, 281-283.

2. Stern, M. Ueber einige Eigenschaften der Function Ex. J. Reine Angew. Math. 1861, 59, 146-162.

3. Gradshteyn, I.S.; Ryzhik, I.M. Table of Integrals, Series, and Products, 7th ed.; Jeffrey, A., Zwillinger, D., Eds.; Academic Press: New York, NY, USA, 2007.

4. Hansen, E.R. A Table of Series and Products; Prentice Hall: Englewood Cliffs, NJ, USA, 1975.

5. Jolley, L.B.W. Summation of Series; Chapman \& Hall: London, UK, 1925.

6. Prudnikov, A.P.; Brychkov, Y.A.; Marichev, O.I. Integrals and Series Vol. 1. Elementary Functions; Gordon and Breach: New York, NY, USA, 1986.

7. Datta, C.; Agrawal, P.; Choudhary, S.K. Bell Type Inequalities can act as a measure of entanglement. arXiv 2015, arXiv:1503.08495.

8. McCoy, B.M.; Orrick, W.P. Analyticity and integrability in the chiral Potts model. J. Statist. Phys. 1996, 83, 839-865.

9. Fukumoto, $\mathrm{Y}$. The index of the $\operatorname{spin}^{c}$ Dirac operator on the weighted projective space and the reciprocity law of the Fourier-Dedekind sum. J. Math. Anal. Appl. 2005, 309, 674-685.

10. Fukuhara, S. Modular forms, generalized Dedekind symbols and period polynomials. Math. Ann. 1998, 310, 83-101.

11. Kittappa, R. Proof of a conjecture of 1881 on permanents. Linear Multlinear Algebra 1981, 10, 75-82.

12. Minc, H. On a conjecture of R.F. Scott (1881). Linear Algebra Appl. 1979, 28, 141-153.

13. Chu, W.; Marini, A. Partial fractions and trigonometric identities. Adv. Appl. Math. 1999, 23, 115-175.

14. Berndt, B.C.; Yeap, B.P. Explicit evaluations and reciprocity theorems for finite trigonometric sums. Adv. Appl. Math. 2002, 29, 358-385.

15. Brown, J.W.; Churchill, R.V. Complex Variables and Applications, 6th ed.; McGraw-Hill, Inc.: New York, NY, USA, 1996.

16. Apostol, T.M. On the Lerch Zeta Function. Pacific J. Math. 1951, 1, 161-167.

(C) 2017 by the authors; licensee MDPI, Basel, Switzerland. This article is an open access article distributed under the terms and conditions of the Creative Commons Attribution (CC BY) license (http:/ / creativecommons.org/licenses/by/4.0/). 